\title{
The effect of a gamma ray flare on Schumann resonances
}

\author{
A. P. Nickolaenko ${ }^{1}$, I. G. Kudintseva ${ }^{2}$, O. Pechony ${ }^{3}$, M. Hayakawa ${ }^{4}$, Y. Hobara ${ }^{5}$, and Y. T. Tanaka ${ }^{6}$ \\ ${ }^{1}$ Usikov Institute for Radio-Physics and Electronics, National Academy of Sciences of the Ukraine, \\ 12, Acad. Proskura Street, Kharkov 61085, Ukraine \\ ${ }^{2}$ Karasin Kharkov National University, 4, Svoboda sq., Kharkov 61077, Ukraine \\ ${ }^{3}$ NASA Goddard Institute for Space Studies and Columbia University, New York, NY, USA \\ ${ }^{4}$ Advanced Wireless Communications Research Center and Research Station on Seismo Electromagnetics, The University of \\ Electro-Communications 1-5-1 Chofugaoka, Chofu, Tokyo 182-8585, Japan \\ ${ }^{5}$ The University of Electro-Communications, Graduate School of Informatics and Engineering, 1-5-1 Chofugaoka, Chofu \\ Tokyo 182-8585, Japan \\ ${ }^{6}$ Institute of Space and Astronautical Science, Japan Aerospace Exploration Agency, Sagamihara, Kanagawa, 229-8510, Japan \\ Correspondence to: M. Hayakawa (hayakawa@whistler.ee.uec.ac.jp)
}

Received: 12 November 2010 - Revised: 21 August 2012 - Accepted: 22 August 2012 - Published: 7 September 2012

\begin{abstract}
We describe the ionospheric modification by the SGR 1806-20 gamma flare (27 December 2004) seen in the global electromagnetic (Schumann) resonance. The gamma rays lowered the ionosphere over the dayside of the globe and modified the Schumann resonance spectra. We present the extremely low frequency (ELF) data monitored at the Moshiri observatory, Japan $\left(44.365^{\circ} \mathrm{N}, 142.24^{\circ} \mathrm{E}\right)$. Records are compared with the expected modifications, which facilitate detection of the simultaneous abrupt change in the dynamic resonance pattern of the experimental record. The gamma flare modified the current of the global electric circuit and thus caused the "parametric" ELF transient. Model results are compared with observations enabling evaluation of changes in the global electric circuit.
\end{abstract}

Keywords. Electromagnetics (Electromagnetic theory; Wave propagation)

\section{Introduction}

Monitoring of the global electromagnetic (Schumann) resonance allows for studying both the Earth-ionosphere cavity and the natural sources of radiation-lightning strokes (Nickolaenko and Hayakawa, 2002). We compare the experimental and model results concerning the impact of the powerful gamma ray flare from SGR 1806-20 that occurred on 27 December 2004. Since 1979 a couple of intense gamma ray flares took place arriving from the extra-terrestrial sources.
Records of remote VLF transmitters indicated the ionosphere depression caused by the gamma rays (Inan et al., 1999, 2007; Terasawa et al., 2005; Tanaka et al., 2008), but an attempt was unsuccessful at finding any changes in the Schumann resonance records caused by the gamma flare from SGR 1900+14 on 27 August 1998 (Price and Mushtak, 2001).

In this paper we report a successful detection of changes in the Schumann resonance spectra during the intense gamma ray flare SGR 1806-20 (27 December 2004). The records were performed at the Moshiri observatory, Japan $\left(44.365^{\circ} \mathrm{N}\right.$ and $142.24^{\circ} \mathrm{E}$ ). Experimental material is compared with the model predictions for the Schumann resonance background signal based on the "knee" ionosphere model (Mushtak and Williams, 2002; Williams, et al., 2006), and the partially uniform knee (PUK) ionosphere model (Pechony and Price, 2004). We also compare the experimental records at the Moshiri observatory with the model estimates for the parametric ELF pulse generated by the gamma flare (Nickolaenko, 2010, 2011; Nickolaenko and Schekotov, 2011a, b).

Detailed description of the gamma ray event can be found in the papers by Hurley et al. (2005), Terasawa et al. (2005), Inan et al. (1999, 2007), and Tanaka et al. (2011). We mention only the minimal information here. The flare occurred around 21:30:26 UT when the hard X/gamma rays arrived at the dayside of the Earth. Radiation came from a neutron star 30-40 thousand light years away. The peak flow exceeded the most intense solar flares by five orders of magnitude, and it was 100 times greater than the $S G R$ 1900+14 gamma flare of 
1998. The disturbance was centered above the Pacific Ocean $\left(146.2^{\circ} \mathrm{W}\right.$ and $\left.20.4^{\circ} \mathrm{S}\right)$, i.e. at a distance of $\approx 450 \mathrm{~km}$ from the center of the dayside hemisphere.

Monitoring the transpacific VLF transmissions at the "Palmer" Antarctic station allowed for the following interpretation of observations (Inan et al., 2007). The gamma flare lowered the dayside ionosphere by $\sim 20 \mathrm{~km}$, and modification lasted for more than $1 \mathrm{~h}$. It was detected at distances up to $60^{\circ}$ from the sub-flare point. An abrupt drop of the ionosphere height occurred in less than $0.02 \mathrm{~s}$ (Inan et al., 2007), and we fit the temporal change of the height by the following function:

$d H=-19 \cdot(9.6441 t)^{-0.1501}$.

Here $t$ is the time since the gamma ray arrival. In our computations, we change the ionosphere height with a $10 \mathrm{~s} \mathrm{step}$ (Nickolaenko and Hayakawa, 2010).

\section{Conductivity profile of the lower ionosphere}

The knee ionosphere model is used for computing the parameters of Schumann resonances in the uniform Earthionosphere cavity (Williams et al., 2006). The PUK model is applied for the cavity with day-night non-uniformity (Price and Pechony, 2004). We postulate that only the "knee altitude" is reduced by gamma rays. Therefore, the "electric" height of the Earth-ionosphere cavity goes down, while the "magnetic" height remains undisturbed. It is important that according to VLF records (Inan et al., 2007), the modification was detected at the angular distance $\psi$ from the center of disturbance reaching $60^{\circ}$. We assume therefore that the gamma ray modification may cover the whole hemisphere.

Physically, a huge reduction of the ionosphere height alters the eigenvalues of the Earth-ionosphere cavity. Modification simultaneously brings down the peak amplitudes, peak frequencies, and the quality factors of all Schumann resonance modes. In our computations that account for the daynight non-uniformity, the PUK model was used (Pechony and Price, 2004) with the $20 \mathrm{~km}$ reduction of knee height at the dayside. Bearing in mind the global nature of Schumann resonance, we applied the $10 \mathrm{~km}$ depression over the whole globe in the model of uniform Earth-ionosphere cavity (Nickolaenko and Hayakawa, 2010). We compare the results of both approaches and find that the day-night asymmetry has a minor impact on the quantitative effect of the gamma rays.

\section{Modifications of Schumann resonance spectra}

Prior to processing the experimental record, we found out what kind of modification should be sought in the observational data (Nickolaenko and Hayakawa, 2010). The above models of the Earth-ionosphere cavity were used for this purpose combined with the modern data on the global lightning source distribution. The "physical" parameters of the standard and disturbed ionospheres are listed below.

We adopt the knee altitude $H_{\mathrm{KNEE}}=55 \mathrm{~km}$ for the regular model, and the knee frequency $F_{\mathrm{KNEE}}=10 \mathrm{~Hz}$. This means that conductivity and displacement currents become equal $\left(2 \pi \cdot \varepsilon_{0} \cdot F_{\mathrm{KNEE}}=\sigma_{\mathrm{KNEE}}\right)$ at the $55 \mathrm{~km}$ altitude for the frequency of $10 \mathrm{~Hz}$ (Greifinger and Greifinger, 1978; Kirillov, 1993, 1996; Kirillov and Kopeykin, 2002; Mushtak and Williams, 2002; Williams et al., 2006; Pechony et al., 2007). Vertical conductivity profile has the scale heights $\zeta_{\mathrm{a}}=2.9 \mathrm{~km}$ above and $\zeta_{\mathrm{b}}=8.3 \mathrm{~km}$ below the knee altitude. The upper ionosphere is characterized by the magnetic height $H_{\mathrm{mp}}=96.5 \mathrm{~km}$ and $F_{\mathrm{mp}}=8 \mathrm{~Hz}$, which logarithmically varies with frequency. Conductivity profile at the "magnetic" altitude has a definite scale height (Mushtak and Williams, 2002; Williams at al., 2006). Gamma rays globally reduce the knee altitude $H_{\mathrm{KNEE}}$ from 55 to $45 \mathrm{~km}$, and relevant parameters were used in computations of ELF spectra (Nickolaenko and Hayakawa, 2010).

When accounting for the cavity day-night asymmetry, we used the PUK model (Pechony and Price, 2004; Pechony et al., 2007). The ionosphere is subdivided into the day and night hemispheres, and the knee model is used for each of them, only, parameters at these two sides are different. In particular, $F_{\mathrm{KNEE}}=13 \mathrm{~Hz}$ and $F_{\mathrm{mp}}=6 \mathrm{~Hz}$ are fixed everywhere. The dayside is characterized by $H_{\mathrm{KNEE}}=54 \mathrm{~km}$, $\zeta_{\mathrm{a}}=2.7 \mathrm{~km}, \zeta_{\mathrm{b}}=7.5 \mathrm{~km}$, and $H_{\mathrm{mp}}=97.5 \mathrm{~km}$. The nightside has the values of $H_{\mathrm{KNEE}}=60 \mathrm{~km}, \zeta_{\mathrm{a}}=3.8 \mathrm{~km}, \zeta_{\mathrm{b}}=$ $9.1 \mathrm{~km}$, and $H_{\mathrm{mp}}=99.0 \mathrm{~km}$. These parameters were used in the procedure of resolving the 2-D (two-dimensional) telegraph equations for Schumann resonance.

Two spatial distributions of thunderstorms were used. The simple one has the lightning strokes uniformly distributed worldwide. A more realistic model implies the global distribution acquired by the Optical Transient Detector (OTD). This space-borne sensor recorded optical flashes from the lightning strokes worldwide during 5 years of observations. The final results were presented as global maps of lighting activity corresponding to every hour UT and each month of a year. Since the gamma rays arrived at 21:30 UT, we averaged the OTD maps for 21 and $22 \mathrm{~h}$ in our computations.

Figure 1 shows the numerical sonogram of Schumann resonance around the moment of gamma ray burst $(t=0)$ (Nickolaenko and Hayakawa, 2010). Thunderstorms were positioned in accordance with the OTD data. Resonance spectra were computed with the $10 \mathrm{~s}$ time step, and the height modification was described by Eq. (1) with the initial global reduction of $10 \mathrm{~km}$ instead of $19 \mathrm{~km}$.

The sonogram of Fig. 1 clearly shows an abrupt downward shift of the Schumann resonance pattern at the onset of gamma ray flare. It slowly returns to the regular configuration afterwards. Thus, one has to search for a sudden drop in experimental sonograms at the time of the gamma burst occurrence. To stress the outline of modification, we draw the 


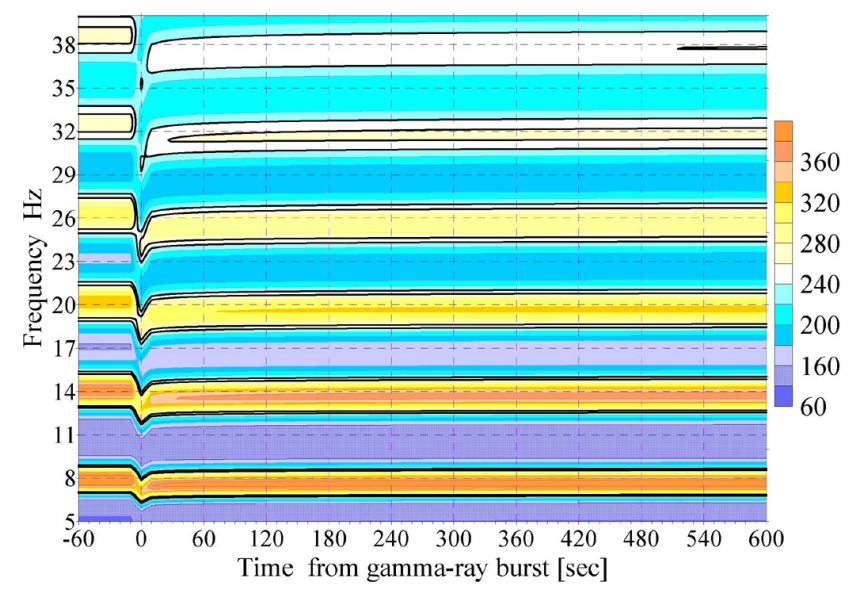

Fig. 1. Modifications in the Schumann resonance spectra produced by the extra-terrestrial gamma ray flare on 27 December 2004 .

levels 240 and 260 a.u. by thick lines in Fig. 1. These contours will be helpful in comparing observational data.

Figure 2 shows the impact of gamma rays in both the uniform Earth-ionosphere cavity (upper plot) and in the nonuniform cavity (lower plot). The OTD source distribution is used in both cases. The spectra were computed for the vertical electric field component by using the 2-D telegraph equations with the PUK day-night model (Pechony et al., 2007). As one may see from the figure, dynamic spectra of Schumann resonance in both frames behave similarly: there is an abrupt drop in the resonance pattern.

Additional data are given in Fig. 3 comparing amplitude variations in the uniform and non-uniform Earth-ionosphere cavities. The observer is placed at "Palmer" Antarctic station where the gamma flare was detected in VLF transmissions. Both uniform and OTD spatial distributions of lightning strokes were used combined with two models of the Earth-ionosphere cavity.

The inset in Fig. 3 shows the amplitude reduction with higher temporal resolution. By comparing the black line (OTD lightning distribution in the non-uniform cavity) with the blue curve (OTD distribution and the uniform cavity), we observe that effect of the ionosphere day-night asymmetry is insignificant. The highest deviation among the curves is pertinent to the uniform distribution of lightning strokes. Except for this systematic deviation, the general behavior remains the same: an abrupt reduction reaching $30 \%$ and a gradual recovery. We conclude that computations predict essentially the same modification regardless of particular model: gamma rays abruptly reduce all resonance parameters. It is important that sudden alterations occur simultaneously at all resonance modes, and hence we must look for the similar discontinuity pattern in experimental ELF sonograms around the occurrence time of gamma flare.

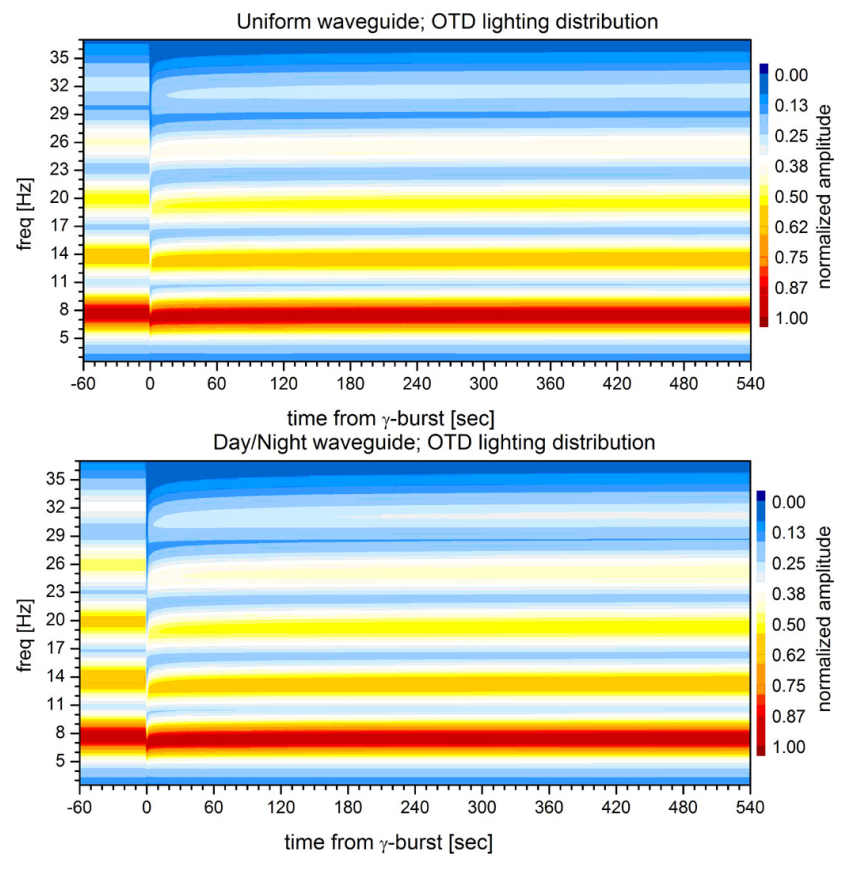

Fig. 2. A comparison of Schumann resonance modifications produced by the gamma ray flare in the uniform cavity and in the nonuniform cavity with the day-night asymmetry.

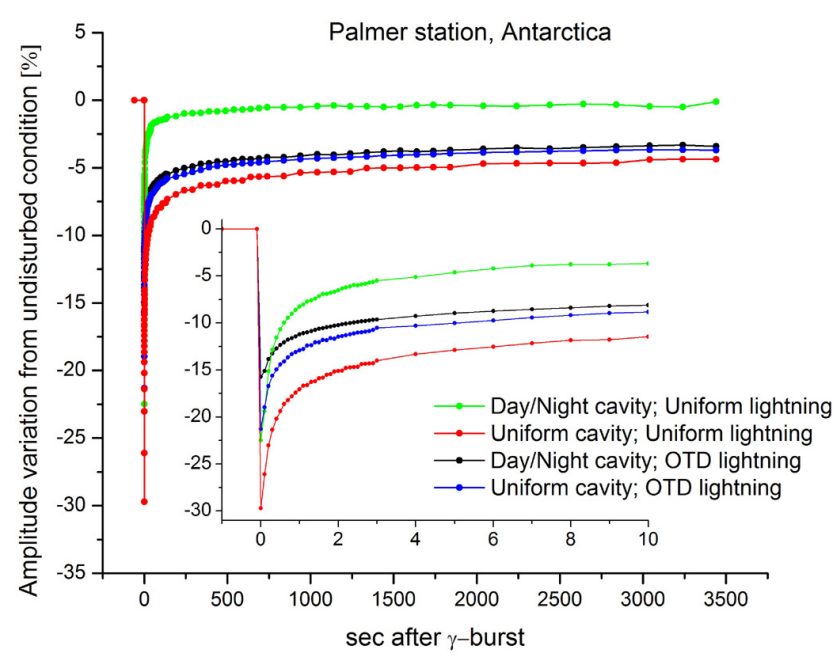

Fig. 3. Modulation of Schumann resonance amplitude by the gamma ray flare in uniform and non-uniform cavities with uniform and OTD spatial source distributions.

\section{Experimental Schumann resonance background signal}

Experimental ELF records are made continuously at the Moshiri observatory, Japan (geographic coordinates, $44.365^{\circ} \mathrm{N}$ and $142.24^{\circ} \mathrm{E}$ ), since 1996 . Waveforms of vertical electric, east-west (EW), and north-south (NS) horizontal magnetic field components are recorded here with the 

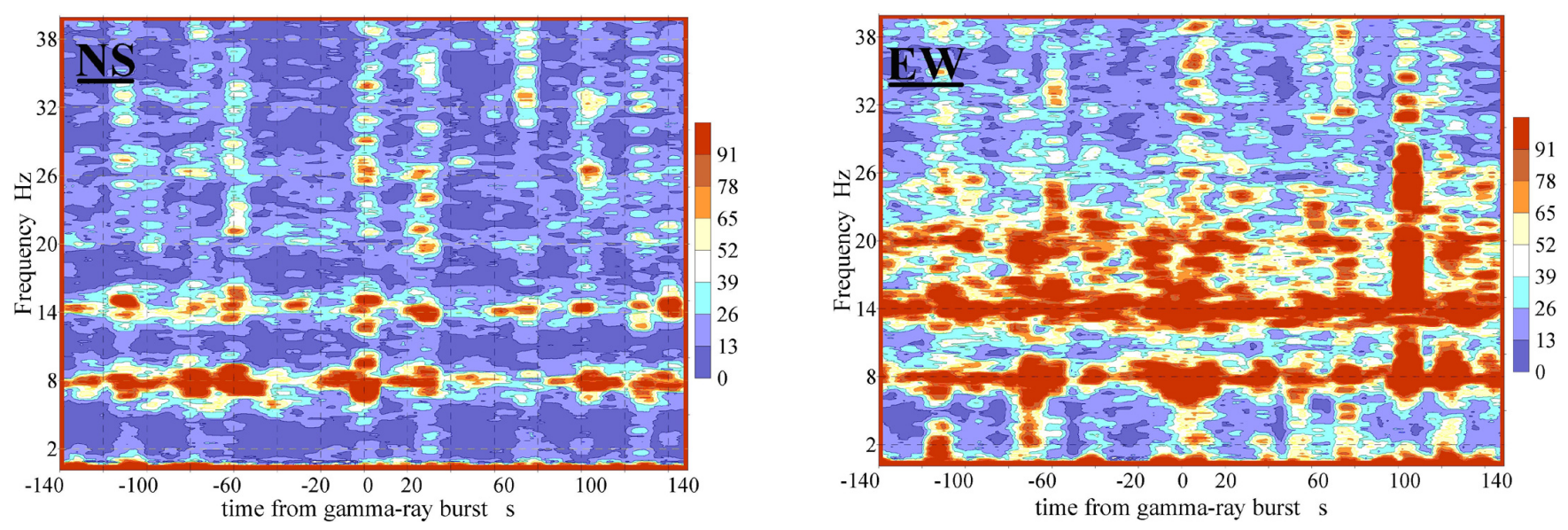

Fig. 4. Survey sonograms of Schumann resonance records at Moshiri observatory around the time of the gamma ray flare.

sampling frequency of $4 \mathrm{kHz}$ (Ando et al., 2005). The ELF data at Moshiri have been utilized to study the sprite problems (Hobara et al., 2001; Hayakawa et al., 2004), global lighting activity (Sekiguchi et al., 2006), etc. (see a review by Hayakawa et al., 2011). We have chosen a fragment of the ELF record in the vicinity of the gamma ray event for the spectral processing. Dynamic spectra of $H_{\mathrm{NS}}$ and $H_{\mathrm{EW}}$ components are shown in Fig. 4 (the electric signal was spoiled by local interference). We depict two survey sonograms in this figure. Signal frequency is plotted on the ordinate in $\mathrm{Hz}$. Time is shown on the abscissa in seconds, and the zero time is linked to the moment of gamma ray arrival. Individual amplitude spectra composing the sonograms were obtained from fragments $10 \mathrm{~s}$ long. Such a duration provided the frequency resolution of $0.1 \mathrm{~Hz}$. Individual spectra were obtained with a $1 \mathrm{~s}$ step, i.e. for $t=0$; we process the segment ranging from -5 to $+5 \mathrm{~s}$. For the time $t=1$, the segment was $[-4 ; 6 \mathrm{~s}]$, etc. Thus, the time shown on the abscissa corresponds to the middle of a record $10 \mathrm{~s}$ long.

It is well known that Schumann resonance spectra of short samples of data are rather unstable (e.g. Nickolaenko and Hayakawa, 2002). The commonly known smooth resonance lines appear after averaging of many elementary spectra, and usually the 5 or $10 \mathrm{~min}$ intervals are used for averaging ( 30 or 60 elementary spectra). One cannot expect the "regular" experimental resonance pattern like those shown in Figs. 1 and 2: observational sonograms are highly structured due to the stochastic nature of lightning sources. One may see that spectra in Fig. 4 are spontaneous in both the field components. The outline of the gamma ray modification is obscured by random changes driven by the global thunderstorms. In this aspect our experimental spectra are similar to data published by Price and Mushtak (2001).

To reveal any modification, we choose a smaller portion of experimental sonograms and combine them in Fig. 5 with the spectra of Fig. 1. The thick black lines depict the levels emphasized in the model sonogram. In spite of highly unstable experimental spectra, the expected simultaneous drop of the resonance pattern becomes apparent in both frames of Fig. 5 . The time of abrupt modification matches the arrival moment of gamma rays to Earth. Even the tendency toward recovery might be perceived in the plots of Fig. 5 .

\section{ELF transient caused by gamma ray flare}

An original simple model of parametric excitation was aimed at evaluating the expected amplitude of a feasible parametric pulse (Nickolaenko, 2010). It suggested that the Earthionosphere cavity is a dielectric spherical layer separating the perfectly conducting ground and ionosphere. The ionosphere is charged to $+250 \mathrm{kV}$ potential, and the gamma flare reduces the regular $60 \mathrm{~km}$ height to $40 \mathrm{~km}$ over the dayside of the globe. Computations predicted the pulsed amplitude exceeding the Schumann resonance background signal by at least an order of magnitude (Nickolaenko, 2010).

After the parametric pulse was discovered in the records of three Schumann resonance observatories (Tanaka et al., 2011), more realistic models were elaborated (Nickolaenko, 2011) for interpreting the observations (Nickolaenko and Schekotov, 2011a, b). One of these models is used in the present work.

Figure 6 depicts the fragment of record, and the rectangular frame denotes the time window when the parametric ELF transient must be expected. The abscissa depicts the time in seconds from 21:30 UT. Three records are shown: the upper one presents the vertical electric field and two lower plots correspond to the $H_{\mathrm{EW}}$ and $H_{\mathrm{NS}}$ horizontal magnetic field components. The electric field was damaged by the local interference at the time and thus was not processed.

Horizontal magnetic fields also contain the man-made interference, which does not completely conceal the ELF transient pulse at the expected time. It was necessary to remove the interference from the record. We used the 

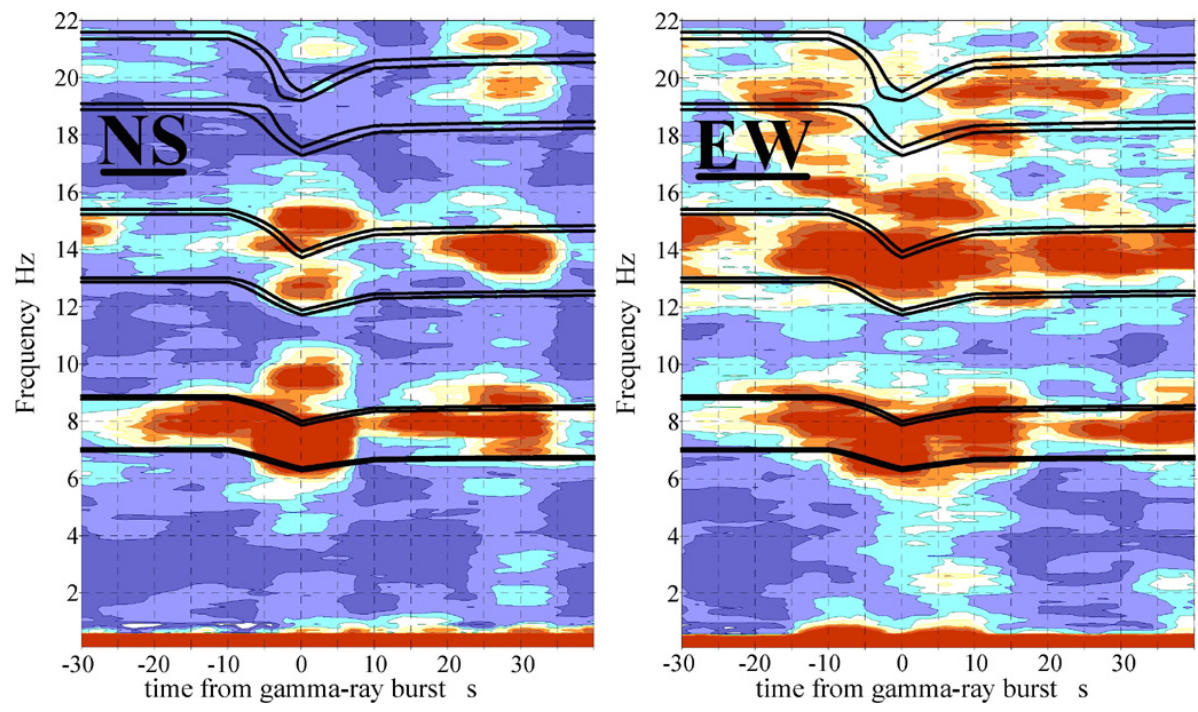

Fig. 5. A comparison of experimental and model sonograms around the time of the gamma ray flare, revealing the Schumann resonance modification.

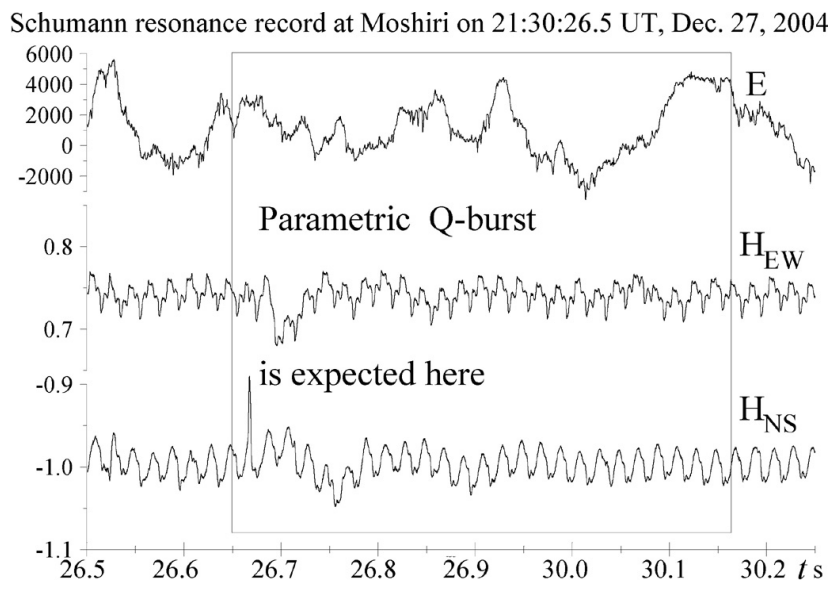

Fig. 6. Fragment of ELF record at Moshiri station around the time of gamma ray flare.

CATERPILLAR algorithm accomplishing the singular spectral analysis (SSA) (Danilov and Zhiglyavsky, 1997; Marple, 1987), and Fig. 7 shows the stages of its application. The left frame in Fig. 7 shows the processing of $H_{\mathrm{NS}}$ field and the right frame corresponds to $H_{\mathrm{EW}}$ component. The abscissa shows the time in seconds from 21:30 UT.

The upper plots show the original record. The second graph in the left frame of Fig. 7 illustrates the sum of principal components \#1, \#2, and \#3 corresponding to the pulsed and high frequency interference in the $H_{\mathrm{NS}}$ record. After removing it from the initial realization, we obtain the third plot of Fig. 7 (single CATERPILLAR procedure). A subsequent application of the same algorithm removes the remaining $50 \mathrm{~Hz}$ signal completely, and we obtain the lower red curve in Fig. 7. This is an attenuating pulse of $\sim 7 \mathrm{~Hz}$ "carrier" frequency.

In processing of the $H_{\mathrm{EW}}$ field component (the right frame of Fig. 7), the complicated industrial interference is recognized as the principal component \#1 (the second plot). It is readily removed, and we obtain the pulsed waveform of $\sim 7 \mathrm{~Hz}$ frequency. Pulses "purified" by the SSA processing were used in the spectral analysis exploiting the FFT algorithm. Relevant amplitude spectra are shown in Fig. 8. Here, the abscissa depicts the signal frequency in $\mathrm{Hz}$, and the field amplitude is shown on the ordinate in arbitrary units. The inset in Fig. 8 demonstrates the Schumann resonance band in more detail. One may observe that pulses in the both field components have the distinct first Schumann resonance mode, while the $H_{\mathrm{EW}}$ component also contains a weak and wide second mode.

We depicted in Fig. 8 the amplitude spectra up to $120 \mathrm{~Hz}$, as the model (Nickolaenko, 2010) predicted two distinct spectral peaks. One of them was the first Schumann resonance mode being a sole maximum in the spectrum due to source current covering the whole hemisphere. Physically, individual "sub-signals" arriving from different sections of a large parametric source constructively interfere only at the lowest resonance frequency. The signals interfere destructively at higher modes. The additional maximum in the model spectra was a wide peak around $60 \mathrm{~Hz}$, here, the constructive interference reappears again owing to the $2 \pi$ spatial phase shift. Such a peak was present in the model spectra, but it was absent in all experimental records.

Explanation of the fact is a smaller sector covered by the parametric currents. Computational results for different source widths are shown in Fig. 9 for the Moshiri observatory. One may observe that the "high frequency" peak 

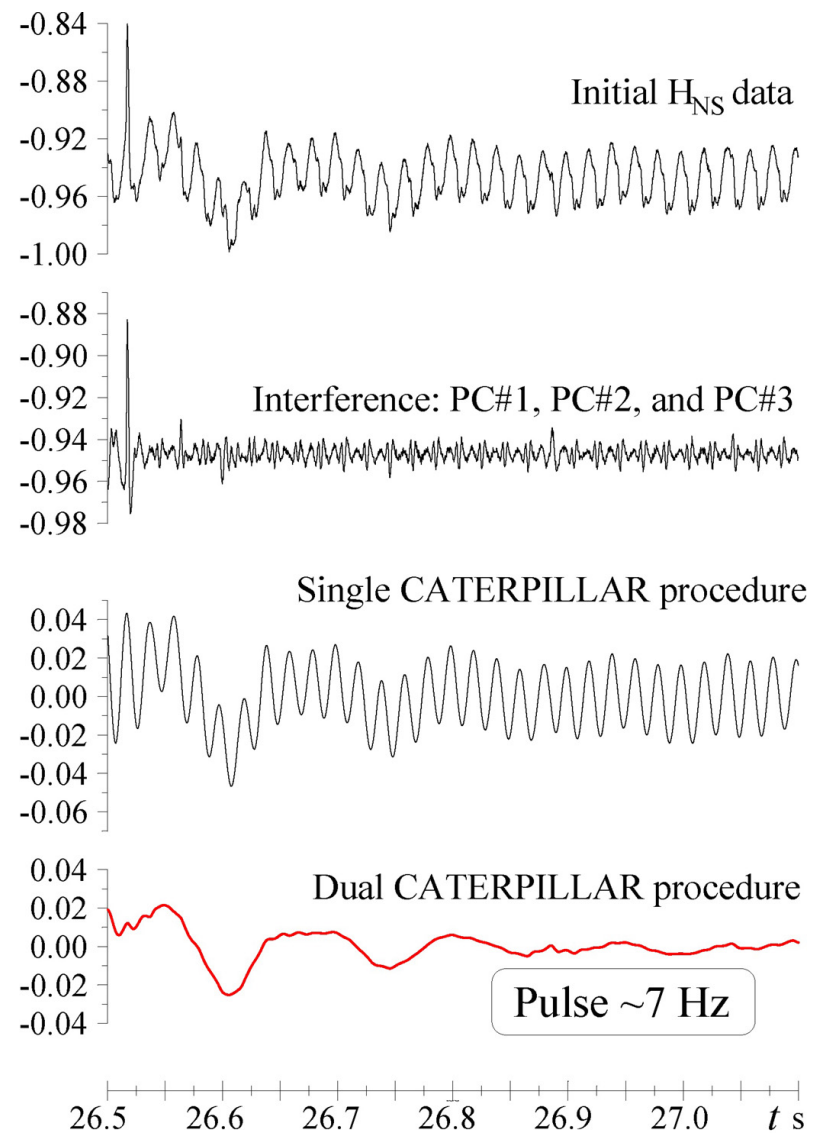

Fig. 7. Preliminary processing of Moshiri experimental record.

disappears in all field components when the source radius is $\psi=60-70^{\circ}$ or smaller. Thus, observations might attest that radius of the area involved in the parametric pulse generation did not surpass the $7 \mathrm{Mm}$. This is in accord with the VLF observations (Inan et al., 2007).

\section{Properties of experimental ELF pulse}

The parametric ELF transient signal (Q-burst) was successfully found in the experimental record. Some of its features coincide with the predictions; some of them deviate from expectations. The occurrence of parametric pulse is consistent with the time of ionosphere modification, and the temporal correspondence is obvious from Fig. 6. Similar pulses were found worldwide in the records of Schumann resonance observatories at Onagawa, Japan $\left(38.43^{\circ} \mathrm{N}, 141.48^{\circ} \mathrm{E}\right)$; Esrange, Sweden, $\left(67.83^{\circ} \mathrm{N}, 21.1^{\circ} \mathrm{E}\right)$; and Karymshino, Russia $\left(52.94^{\circ} \mathrm{N}, 158.25^{\circ} \mathrm{E}\right)$ (Tanaka et al., 2011; Nickolaenko and Schekotov, 2011a, b).

After obtaining information confirming the existence of parametric pulse, advanced models were elaborated for the transient sources (Nickolaenko, 2011). These suggest that the gamma flare modifies the currents of the global electric cir-

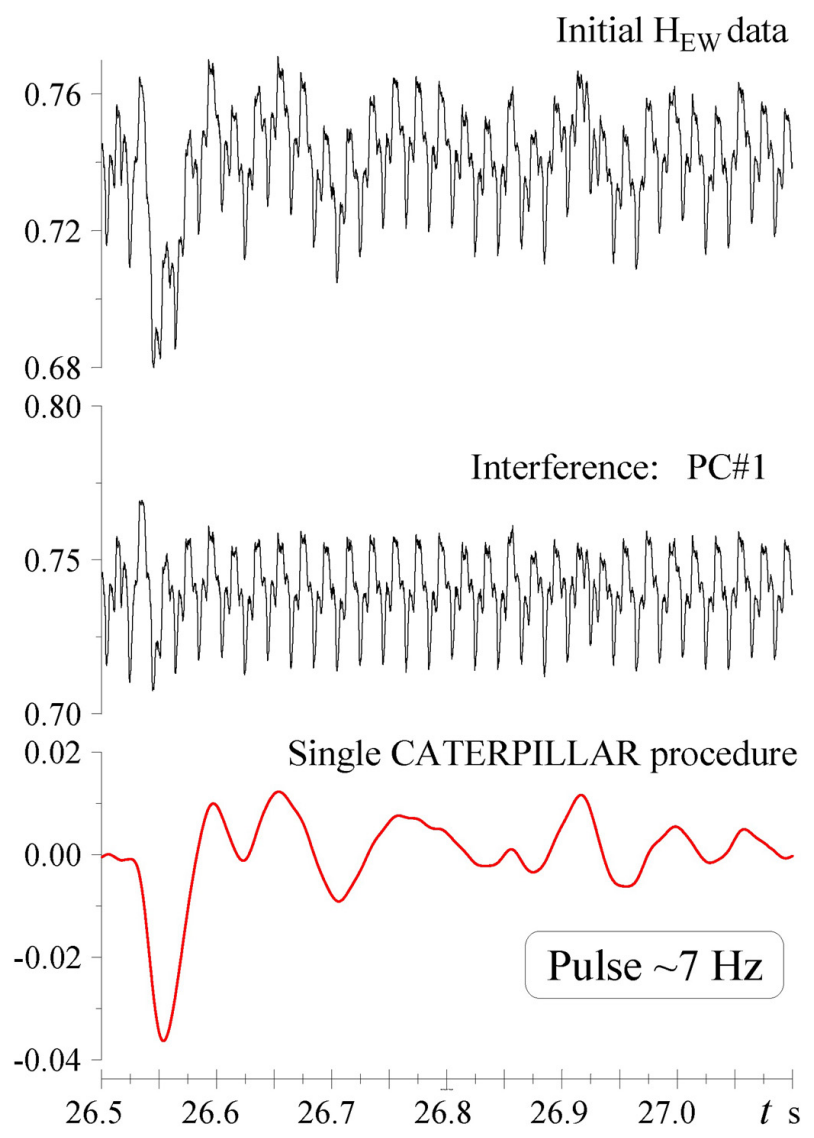

cuit, as an additional ionization increases the air conductivity from the ionosphere heights to the ground. In the specific model we use here, the leakage current from the ionosphere to the ground increases from its regular $2 \mathrm{pA} \mathrm{m}^{-2}$ value to $\sim 4 \mathrm{pA} \mathrm{m}^{-2}$. This change occurs at the moment when gamma rays reach the "electrosphere" heights. The latter is the altitude interval where the major resistance of air column is found (Rycroft et al., 2008). The "proportional-knee" model was used containing the bi-exponential undisturbed vertical profile of air resistance characterized by scale heights of $\zeta_{\mathrm{a}}=2 \mathrm{~km}$ above and $\zeta_{\mathrm{b}}=6 \mathrm{~km}$ below the $55 \mathrm{~km}$ knee altitude (Williams, 2006). The gamma flare moves downward both the knee altitude and the upper part of the profile by $20 \mathrm{~km}$ with no impact on its scale height. The lower part of disturbed vertical profile acquires the new scale height of $\zeta \approx$ $3.5 \mathrm{~km}$, so that particular air resistance at the ground does not vary: $\rho_{0}=2.08 \times 10^{14} \mathrm{Ohm} \mathrm{m}^{-3}$ (Nickolaenko, 2011). The step-like modulation in the leakage current of the global electric circuit provides a "red" parametric source having the $1 / f$ spectral amplitude in distinction from the "white" source spectrum used in the primary model. Since the positive leakage current increases, the source has the positive polarity.

Two features are responsible for an absence of the $60 \mathrm{~Hz}$ peak. The obvious reason is the $1 / f$ source amplitude 


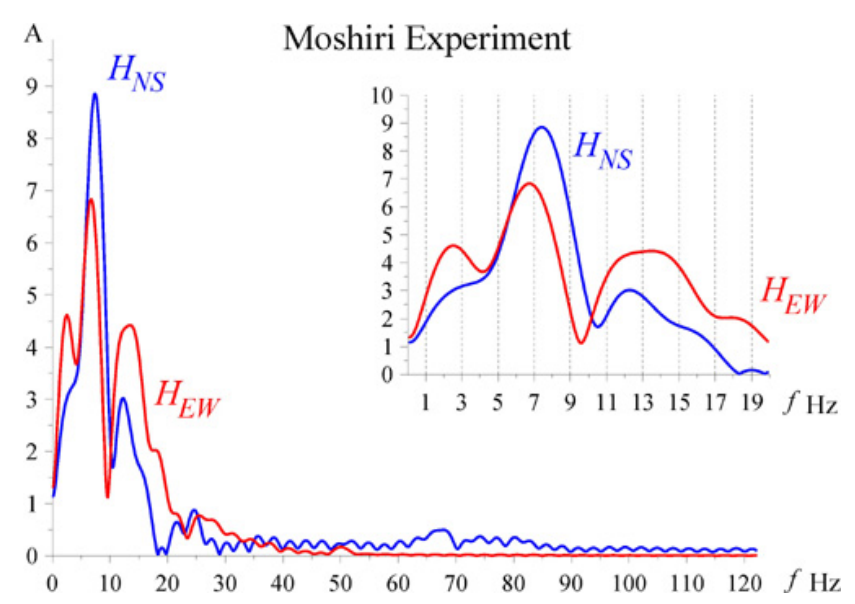

Fig. 8. Amplitude spectra of the pre-processed ELF pulses recorded at the Moshiri observatory around the time of the galactic gamma burst.

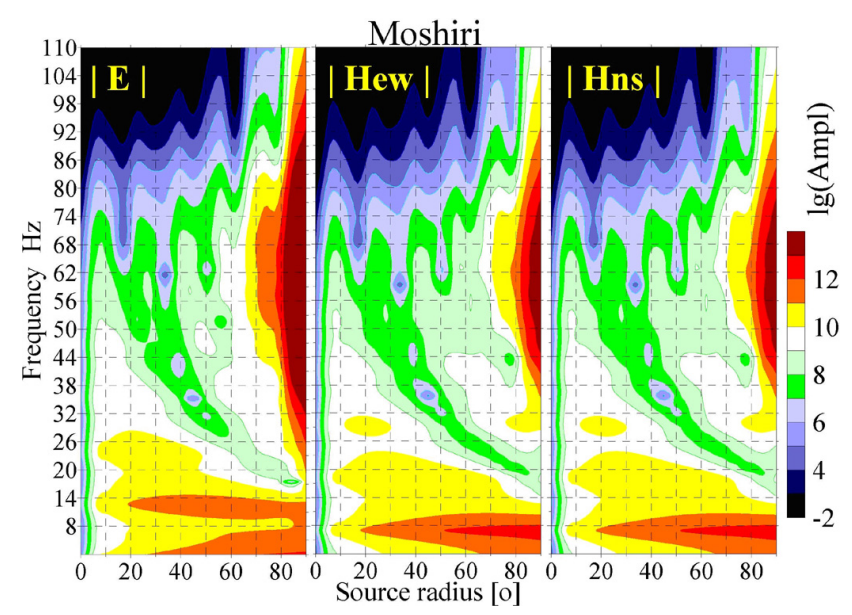

Fig. 9. Spectra of the parametric ELF pulse at Moshiri observatory versus the source width.

spectrum. The $60 \mathrm{~Hz}$ peak becomes noticeably reduced, but "remnants" of the high frequency pattern are still visible in the pulsed waveform. High frequencies disappear completely when the source size becomes equal to $60^{\circ}$ or less (see Fig. 9).

We compare in Fig. 10 the experimental and model waveforms. The abscissa depicts the time in seconds. The pulse amplitude is measured in $\mu \mathrm{Am}^{-1}\left(1 \mu \mathrm{Am}^{-1}=1.256 \mathrm{pT}\right)$. The red line depicts the experimental $H_{\mathrm{NS}}(t)$ waveform, while the blue line shows the model data. As one may observe, waveforms have similar outline and coincident amplitudes. Pulses in Fig. 10 have similar "precursors" and the onsets of the same sign, hence the currents causing the parametric pulse, had the positive polarity, i.e. similar to those of the positive lightning strokes.

Data presented here agree with the records of other stations (Tanaka, 2011; Nickolaenko and Schekotov, 2011a, b).

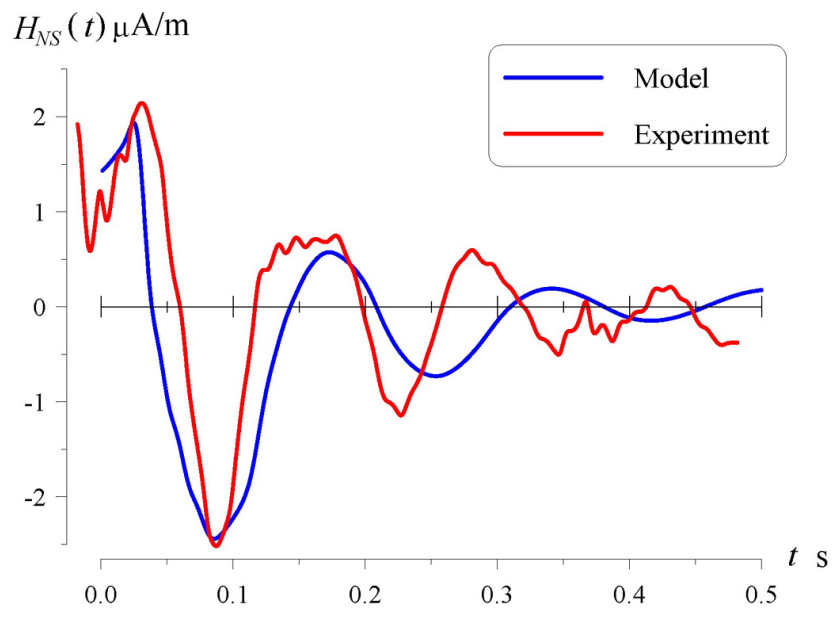

Fig. 10. Experimental (red) and computed (blue) waveforms of $H_{\mathrm{NS}}$ field component.

Observations confirmed the global detection of a parametric Q-burst generated by the gamma ray flare: the pulse arrival times agree with the arrival of gamma quanta, the basic Schumann resonance mode dominates in all records, the observed and computed pulse amplitudes match, and the source polarity was positive.

We hope to perform a comparative analysis of all available data aiming at obtaining thorough information on the field source produced by an interaction of powerful ionizing radiation and atmosphere.

\section{Discussion and conclusion}

Both the model and experimental data presented here show an anomaly in the Schumann resonance pattern caused by the powerful gamma ray flare of 27 December 2004. To detect modifications in the ELF background signal, we had to apply a special signal processing: the $10 \mathrm{~s}$ segments were used in the FFT procedure, while the sonogram was formed by amplitude spectra obtained with the $1 \mathrm{~s}$ step. Owing to the stochastic nature of the experimental record, only its direct comparison with the model sonograms enabled disclosing the expected modification in the spectral pattern.

These results agree with the conclusion by Mushtak and Williams (2002): the random nature of sources results in the stochastic spectra, which impede detection of the short-term gamma ray effect. Usually, Schumann resonance spectra are averaged over the 5-10 min intervals, while the ELF impact of gamma rays becomes indistinguishable in a few seconds after its onset. The unsuccessful attempt by Mushtak and Williams (2002) might be attributed to the smaller intensity of the gamma ray event in 1998 and to the application of a standard averaging procedure.

Ionospheric modification was quite obvious in the records of VLF radio transmitters (Inan et al., 2007). Probably, it 
would be also clearly seen in the records of ELF transmitters (Yano et al., 2010). Concerning Schumann resonances, the effect is masked by the random nature of global lightning. In this context, it would be desirable to similarly analyze all available records and compare experimental sonograms with the model computations: this task outlines the area of our future work.

The source polarity of an ELF pulse is readily determined by the sign of its onset in the record of vertical electric field $E(t)$. It is positive when the onset is negative. However, we have only the horizontal magnetic field components available, so that establishing the source polarity was more complicated. We identified it by comparing the observed and the model waveforms in the field components $H_{\mathrm{x}}(t)$ and $H_{\mathrm{y}}(t)$. As we noted above, the model used was of an instant increase in the atmospheric conductivity over the dayside of the globe caused by the arrival of gamma rays. This leads to an abrupt growth of the leakage current from the ionosphere to the ground in the atmosphere around the sub-flare point. Such a modification has the current moment similar to the positive lightning stroke. Coincidence of the signs of the model and observed pulses indicates the positive source polarity (see Fig. 10). Concerning the sign (polarity) in the experimental record, it depends on the correctness of the coil connection, which was checked carefully in many experiments conducted with these antennas (e.g. Hayakawa et al., 2004; Hobara et al., 2006). A special publication was made that compared different techniques of the source location by using the parent lightning discharges with known positions and polarities (Nakamura et al., 2010). This is why we are sure about the positive source polarity without resort to the vertical electric field measurement.

There is an alternative explanation of the origin of a parametric pulse current, which was put forward by the reviewer of this article, E. R. Williams. The reviewer suggested that the parametric source was formed by the flux of Compton electrons in the atmosphere generated by the cosmic gamma rays. The similar model was used when interpreting the huge ELF pulse produced by the high-altitude thermonuclear explosion "Starfish" (Gendrin and Stefant, 1962). However, such a model contradicts to the observations, because the polarity of the Compton source is negative. Parametric pulses coming from such a source have the onsets of opposite sign in comparison with the observations. To have the consistence in the sign between the model and observation, one has to center the Compton parametric source at the antipode of the sub-flare point $\left(20.4^{\circ} \mathrm{N}\right.$ and $\left.23.8^{\circ} \mathrm{E}\right)$. However, this is the opposite side of the Earth, which was not illuminated by the gamma rays. Thus, the model of Compton electrons contradicts to our particular observations, and therefore it must be declined.

Summarizing the discussion, we note that the model of a sudden two-fold decrease in the conductivity of the air slab, which was caused by galactic gamma rays, correctly de- scribes both the waveform and the amplitude of a parametric pulse.

The observational results presented allow for the following conclusions:

- An instant decrease of all Schumann resonance parameters occurred at the moment of a sharp ionosphere reduction over the dayside of the globe. The onset of modification was coincident with the moment of gamma ray arrival, and shortly afterwards the resonance pattern has recovered.

- The short-time nature of the event combined with the non-stationary nature of ELF radio noise impeded detection of the effect in usual integrated Schumann resonance spectra. Only direct comparison of experimental and model data revealed the impact of gamma flare.

- The gamma rays modified the current in the global electric circuit, thus causing a discrete ELF radio pulse. The pulse was initially found in the Moshiri record, and it was confirmed by the worldwide records at Onagawa (Japan), Esrange (Sweden), and Karymshino (Russia).

- The spectrum of parametric pulse contains only the first Schumann resonance mode, which is conditioned by a considerable horizontal extension of the source currents.

- The wide spectral peak around $60 \mathrm{~Hz}$ was absent in all records. This indicates that the parametric source radius could not surpass $60^{\circ}-70^{\circ}$.

- A comparison of observed and computed pulsed waveforms supports the concept of a sudden increase in the air conductivity that enhanced the leakage current in the global electric circuit by a factor of two.

Acknowledgements. Editor-in-Chief M. Pinnock and Topical Editor P.-L. Blelly thank E. A. Navarro and E. Williams for their help in evaluating this paper.

\section{References}

Ando, Y., Maltsev, P., Sukhynyuk, A., Goto, T., Yamauchi, T., Hobara, Y., Sekiguchi, M., Ikegami, Y., Sera, M., Korepanov, V., and Hayakawa, M.: New ELF observation system at Moshiri, Japan and assessment of acquired data, J. Atmos. Electr., 25, 29-39, 2005.

Danilov, D. L. and Zhiglyavsky, A. A. (Eds.): Principal Component of The Time Series: the Caterpillar Method, St.-Petersburg state University, St-Petersburg, Russia, 307 pp., 1997 (in Russian).

Gendrin, R. and Stefant, R.: Effect of high altitude thermonuclear explosion of July 1962 on the resonance in the Earth-ionosphere cavity, experimental results, C.R. Acad. Sci., 255, 2273-2275, 1962 (in French). 
Greifinger, C. and Greifinger, P.: Approximate method for determining ELF eigenvalues in the Earth-ionosphere waveguide, Radio Sci., 13, 831-837, 1978.

Hayakawa, M., Nakamura, T., Hobara, Y., and Williams, E.: Observation of sprites over the Sea of Japan and conditions for lightning-induced sprites in winter, J. Geophys. Res., 109, A01312, doi:10.1029/2003JA009905, 2004.

Hayakawa, M., Nickolaenko, A. P., Shvets, A. V., and Hobara, Y.: Recent studies of Schumann resonance and ELF transients, in: Lightning: Properties, Formation and Types, edited by: Wood, M. D., Nova Sci. Pub., Chapter 3, pp. 39-71, 2011.

Hobara, Y., Iwasaki, N., Hayashida, T., Hayakawa, M., Ohta, K., and Fukunishi, H.: Interrelation between ELF transients and ionospheric disturbances in association with sprites and elves, Geophys. Res. Lett., 28, 935-938, 2001.

Hobara, Y., Hayakawa, M., Williams, E., Boldi, R., and Downes, E.: Location and electrical properties of sprite producing lightning from a single ELF site. In: Fu"llekrug M et al. (ed) Sprites, elves and lightning discharges, NATO Science Series, Springer, Dordrecht, pp. 211-235, 2006.

Hurley, K., Boggs, S. E., Smith, D. M., Duncan, R. C., Lin, R., Zoglauer, A., Krucker, S., Hurford, G., Hudson, H., Wigger, C., Hajdas, W., Thompson, C., Mitrofanov, I., Sanin, A., Boynton, W., Fellows, C., von Kienlin, A., Lichti, G., Rau, A., and Cline, T.: An exceptionally bright flare from SGR $1806-20$ and the origins of short-duration big gamma-ray bursts, Nature, 434, 1098 1103, 2005.

Inan, U. S., Lehtinen, N. G., Lev-Tov, S. J., Johnson, M. P., Bell, T. F., and Hurley, K.: Ionization of the lower ionosphere by $\gamma$-rays from a magnetar: Detection of a low energy (3-10 keV) component, Geophys. Res. Lett., 26, 3357-3360, 1999.

Inan, U. S., Lehtinen, N. G., Moore, R. C., Hurley, K., Boggs, S., Smith, D. M., and Fishman, G. J.: Massive disturbance of the daytime lower ionosphere by the giant $\gamma$-ray flare from magnetar SGR 1806-20, Geophys. Res. Lett., 34, L08103, doi:10.1029/2006GL029145, 2007.

Kirillov, V. V.: Parameters of the Earth-ionosphere waveguide at ELF, Problems of Diffraction and Wave Propagation, 25, 35-52, 1993 (in Russian).

Kirillov, V. V.: Two-dimensional theory of electromagnetic wave propagation in the ELF range in the Earth-ionosphere waveguide, Izv. Vyssh. Uchebn. Zaved Radiophyz., 39, 1103-1112, 1996 (in Russian).

Kirillov, V. V. and Kopeykin, V. N.: Solving a two dimensional telegraph equation with anisotropic parameters, Izv. Vyssh. Uchebn. Zaved Radiophyz., 45, 1011-1024, 2002 (in Russian).

Marple Jr., S. L.: Digital Spectral Analyses with Applications, Prentice-Hall, Englewood Cliffs, New Jersey, 1987.

Mushtak, V. C. and Williams, E. R.: ELF propagation parameters for uniform models of the Earth-ionosphere waveguide, J. Atmos. Solar-Terr. Phys., 64, 1989-2001, 2002.

Nakamura, T., Sekiguchi, M., Hobara, Y., and Hayakawa, M.: A comparison of different source location methods for ELF transients by using the parent lightning discharges with known positions, J. Geophys. Res., 115, A00E39, doi:10.1029/2009JA014992, 2010

Nickolaenko, A. P.: Parametric excitation of an extremely lowfrequency radio pulse by a galactic gamma-ray burst on December 27, 2004, Radiophysics and Quantum Electronics, 53, 235-
245, doi:10.1007/s11141-010-9219-7, 2010.

Nickolaenko, A. P.: Source models for 'parametric' Q-burst, J. Atmos. Electricity, 31, 95-110, 2011.

Nickolaenko, A. P. and Hayakawa, M.: Resonances in the Earthionosphere Cavity, Kluwer Academic Publishers, DordrechtBoston-London, 2002.

Nickolaenko, A. P. and Hayakawa, M.: Model disturbance of Schumann resonance by the SGR 1806-20 $\gamma$-ray flare on December 27, 2004, J. Atmos. Electricity, 30, 1-11, 2010.

Nickolaenko, A. P. and Schekotov, A. Yu.: Experimental detection of ELF transient associated with the gamma ray burst of Dec. 27, 2004, Radiophysics and Quantum Electronics, 54, 2011, doi:10.1007/s11141-011-9268-6, 2011a.

Nickolaenko, A. P. and Schekotov, A. Yu.: ELF Q-burst caused by galactic gamma ray burst, XXX URSI General Assembly, Istanbul Turkey, 13-20 August 2011, Abstract EGH-3, 2011 b.

Pechony, O. and Price, C.: Schumann resonance parameters calculated with a partially uniform knee model on Earth, Venus, Mars, and Titan, Radio Sci., 39, RS5007, doi:10.1029/2004RS003056, 2004.

Pechony, O., Price, C., and Nickolaenko, A. P.: Relative importance of the day-night asymmetry in Schumann resonance amplitude records, Radio Sci., 42, RS2S06, doi:10.1029/2006RS003456, 2007.

Price, C. and Mushtak, V.: The impact of the August 27, 1998, $\gamma$-ray burst on the Schumann resonances, J. Atmos. Solar-Terr. Phys., 63, 1043-1047, 2001.

Rycroft, M. J., Harrison, R. G., Nicoll, K. A., and Mareev, E. A.: An overview of Earth's global electric circuit and atmospheric conductivity, Space Sci. Rev., 137, 83-105, doi:10.1007/s11214008-9368-6, 2008.

Sekiguchi, M., Hayakawa, M., Nickolaenko, A. P., and Hobara, Y.: Evidence on a link between the intensity of Schumann resonance and global surface temperature, Ann. Geophys., 24, 1809-1817, doi:10.5194/angeo-24-1809-2006, 2006.

Tanaka, Y. T., Terasawa, T., Yoshida, M., Horie, T., and Hayakawa, M.: Ionospheric disturbances caused by SGR 1900+14 giant gamma ray flare in 1998: Constraints on the energy spectrum of the flare, J. Geophys. Res., 113, A07307, doi:10.1029/2008JA013119, 2008.

Tanaka, Y. T., Hayakawa, M., Hobara, Y., Nickolaenko, A. P., Yamashita, K., Sato, M., Takahashi, Y., Terasawa, T., and Takahashi, T.: Detection of transient ELF emission caused by the extremely intense cosmic gamma-ray flare of 27 December 2004, Geophys. Res. Lett., 38, L08805, doi:10.1029/2011GL047008, 2011.

Terasawa, T., Tanaka, Y. T., Takei, Y., Kawai, N., Yoshida, A., Nomoto, K., Yoshikawa, I., Saito, Y., Kasaba, Y., Takashima, T., Mukai, T., Noda, H., Murakami, T., Watanabe, K., Muraki, Y., Yokoyama, T., and Hoshino, M.: Repeated injections of energy in the first $600 \mathrm{~ms}$ of the giant flare of SGR 1806-20, Nature, 434, 1110-1111, doi:10.1038/nature03573, 2005.

Williams, E. R., Mushtak, V., and Nickolaenko, A. P.: Distinguishing ionospheric models using Schumann resonance spectra, J. Geophys. Res., 111, D16107, doi:10.1029/2005JD006944, 2006.

Yano, M., Ida, Y., Hobara, Y., Hayakawa, M., and Nickolaenko, A. P.: Reception of ELF transmitter signals at Moshiri, Japan, and their propagation characteristcs, Radio Sci., 45, RS1009, doi:10.1029/2009RS004224, 2010. 